\title{
Ordering and Pricing Strategies for Fresh Products with Multiple Quality Levels Considering Consumer Utility
}

\author{
Peiqi Ma \\ School of Management, Jinan University, Guangzhou, China \\ Email: Page-Mar@foxmail.com
}

Received 9 April 2015; accepted 17 May 2015; published 20 May 2015

Copyright ( 2015 by authors and Scientific Research Publishing Inc.

This work is licensed under the Creative Commons Attribution International License (CC BY). http://creativecommons.org/licenses/by/4.0/

(c) (i) Open Access

\begin{abstract}
In this paper, considering a scenario in which there are two quality levels of fresh products and introduction of consumer utility function, we studied the optimal ordering and pricing strategies under certain quantity. Our results showed that, facing the two quality levels of fresh products, retailers would not benefit from sales of lower quality of fresh products with the deterministic demand. In the pursuit of profit maximization, the initial order quantity is smaller than the potential demand for market.
\end{abstract}

\section{Keywords}

Fresh Products, Consumer Utility, Multiple Quality Level, Pricing and Ordering

\section{Introduction}

Given the technological advancements and logistical capabilities over the last seven decades or so, perishables have become a large part of supermarket retailing sales. For instance, a report by FMI (2009) indicates that of the $\$ 430$ billion plus in US supermarket sales registered for the year $2008,81 \%$ or over $\$ 348$ billion is due to sales of groceries directly related to foods and beverages ( F \& B). And there is no doubt that fresh foods have played a dramatically important role in china.

Motivated by the common practice, retailers usually divide fresh products, which may decay or deteriorate during the sale process, into different quality levels, and sell them in a separate way. In this paper, considering a scenario in which there are two quality levels of fresh products and introduction of consumer utility function, we studied the optimal ordering and pricing strategies under certain quantity. Then, we discussed how environmental factors, such as demand volatility and ordering costs, affected retailers' decision. By considering consumer 
utility to study the optimal ordering and pricing decisions of multi-quality fresh products, we found corresponding answers of the above questions, and gave the management suggestions.

\section{Literature Review}

Ordering and pricing of fresh products are within the scope of supply chain management, which has become a hot issue for discussion and research. Goyal et al. (2001), Li et al. (2010), and Bakker et al. (2012) provided a comprehensive introduction about deteriorating items inventory management research from different perspectives [1]-[3]. Under certain quantity, Xiao et al. (2010) assumed that there are two quality levels of fresh products; retailer balances supply and demand of fresh products with price discount to study the optimal selling strategies, but they do not take characteristics of fresh product into account [4]. Bai and Kendall (2008) proposed a single-period inventory and shelf-space allocation model for fresh produce. The demand rate is assumed to be deterministic and dependent on both the displayed inventory and the items' freshness condition [5]. Avinadav Tal et al. (2009) presents an extension of the classical EOQ model for items with a fixed shelf life and a declining demand rate due to a reduction in the quality of the item in the course of its shelf-life [6]. However, Perishable products are subject to both obsolescence and deterioration, but above researches that study both types of loss are limited. Dan and Chen (2008) created an exponential function with downward slope, trying to denote valuable loss with greenness, and studies the coordination in two-level fresh agricultural supply chain [7]. Cai et al. (2010) considered a supply chain in which a distributor procures from a producer a quantity of a fresh product [8]. During the transportation process, the distributor has to make an appropriate effort to preserve the freshness of the product, and his success in this respect impacts on both the quality and quantity of the product delivered to the market. In reality, each consumer has different preferences to fresh products, which may influence retailer's ordering and pricing strategies. Above two references do not model consumer behavior. Some scholars analyzed pricing decision that involves modeling customer behavior. Ferguson and Koenigsberg (2007) have presented a two-period model where the quality of the leftover inventory is often perceived to be lower by customers, and the firm can decide to carry all, some, or none of the leftover inventory to the next period [9]. This is also a model involving quality drop and quantity change. Akcay et al. (2010) considered a dynamic pricing problem facing a firm that sells given initial inventories of multiple substitutable and perishable products over a finite selling horizon. They modeled this multiproduct dynamic pricing problem as a stochastic dynamic program and analyzed its optimal prices [10]. Li et al. (2012) studied the joint pricing and inventory control problem for perishables when a retailer does not sell new and old inventory at the same time [11]. Sainathan (2013) considered pricing and ordering decisions faced by a retailer selling a perishable product with a two-period shelf life over an infinite horizon [12]. Those scholars considered multiple quality levels of deteriorating or decaying products, however, they are not on the background of fresh products.

The remainder of this article is organized as follows. In Section 2, I review the related literature. In Section 3, I describe demand model and the retailer's profit model, and then I find the solution of base model. In Section 4, I examine how demand affects the retailer's problem. In Section 5, I give a sensitivity analysis for the results.

\section{Base Model}

Considering a scenario in which a single retailer sells one category fresh product throughout the whole sale period with two stages, there may be residual at the end of one stage, and the retailer can sell the low quality product at the next stage. We assumed that there exist two different quality levels (high and low) to discuss effectiveness of retailers selling decision, ordering and pricing strategies. At the start of period, the retailer procure $Q$ unit fresh product with per unit procurement cost of $\omega$ from producer, and the procurement lead time is assumed to be zero. In sales process, retailer can do preservation effort at $\varphi$ rate, $\varphi \in[0,1]$, then its cost is assumed to be $C=\frac{1}{2} Q \varphi$. Then I describe the choice process among customers. Any customer who visits the retailer has three choices: buy one unit of the low quality product, buy one unit of the high quality product, or do not buy anything. The high and low quality products compete among customers in their attributes and prices. Each customer selects his preferred choice based on his utility from purchasing a unit of product typei, which is given by

$$
U_{H}=u \alpha_{H}-P_{H}, U_{L}=u \alpha_{L}-P_{L}
$$


where $u$ is a customer's quality sensitivity, $u \sim U[0,1], \alpha_{i}$ is product typei's quality factor that is a measure of its desirability (therefore $\alpha_{H}>\alpha_{L}$ ), and $P_{i}$ is its price. Then, I define $R_{i}$ to be the probability that a customer buying one unit of the high (low) quality product. Next, I discuss how demand certainty impacts the retailer's optimal strategy

Based on the utility model, I first derive expression for $R_{H}$ and $R_{L}$ as functions of the prices and quality factors. The percentage of a customer buying the high quality level $R_{H}$, and that of buying the old product, $R_{L}$, are as follows:

$$
\begin{gathered}
R_{H}=P_{r}\left\{U_{H} \geq U_{L}, U_{H} \geq 0\right\}=P\left\{u \geq \max \left(\frac{P_{H}}{\alpha_{H}}, \frac{P_{H}-P_{L}}{\alpha_{H}-\alpha_{L}}\right)\right\} \\
R_{L}=P_{r}\left\{U_{L} \geq U_{H}, U_{L} \geq 0\right\}=P\left\{\frac{P_{L}}{\alpha_{L}} \leq u \leq \frac{P_{H}-P_{L}}{\alpha_{H}-\alpha_{L}}\right\}
\end{gathered}
$$

In the expression 2, there is need to compare $\frac{P_{H}}{\alpha_{H}}$ with $\frac{P_{H}-P_{L}}{\alpha_{H}-\alpha_{L}}$, so we have two following cases:

Case 1 When $\frac{P_{H}}{\alpha_{H}}>\frac{P_{H}-P_{L}}{\alpha_{H}-\alpha_{L}}$, i.e. $\frac{P_{H}}{\alpha_{H}}<\frac{P_{L}}{\alpha_{L}}$, which means all customer only buy high quality products. And after it clears out, customer will buy low quality products. Accordingly, we can draw an expression: $R_{H}=P\left\{\frac{P_{H}}{\alpha_{H}} \leq u \leq 1\right\}=1-\frac{P_{H}}{\alpha_{H}}, R_{L}=0$.

Case 2 When $\frac{P_{H}}{\alpha_{H}}<\frac{P_{H}-P_{L}}{\alpha_{H}-\alpha_{L}}$, i.e. $\frac{P_{H}}{\alpha_{H}}>\frac{P_{L}}{\alpha_{L}}$, which means customer will buy fresh products between high and low quality. The expression for $R_{H}$ and $R_{L}$ are then obtained by finding the corresponding probabilities by using the fact that $u \sim U[0,1]$ :

$$
R_{H}=\max \left(1-\frac{P_{H}-P_{L}}{\alpha_{H}-\alpha_{L}}, 0\right), R_{L}=\min \left(\frac{P_{H}-P_{L}}{\alpha_{H}-\alpha_{L}}, 1\right)-\frac{P_{L}}{\alpha_{L}}
$$

Suppose the total number of customers is $m$. The demand for the high and low quality products is deterministic and is given by $m R_{H}$ and $m R_{L}$, respectively. Therefore, the retailer's profit then is given by:

$$
\begin{aligned}
& \pi_{P_{H}, P_{L}}\left(P_{H}, P_{L} \mid M=m, Q\right) \\
& =P_{H} \min \left(m \cdot R_{H}, Q\right)+P_{L} \min \left(m \cdot R_{L},\left(Q-m \cdot R_{H}\right)^{+}\right)-\omega Q-\frac{1}{2} Q \varphi
\end{aligned}
$$

The symbol "+" means the expression, $Q-m \cdot R_{H}$ is positive.

\section{Optimal Ordering and Pricing Decision}

In this section, we solve the optimal ordering and pricing decision according to the retailer's demand and profit model. As the same, there is different relationship between $\frac{P_{H}}{\alpha_{H}}$ and $\frac{P_{H}-P_{L}}{\alpha_{H}-\alpha_{L}}$ when maximizing retailer's profit. So here are two scenarios as following:

Scenario 1: Suppose that $\frac{P_{H}}{\alpha_{H}}>\frac{P_{H}-P_{L}}{\alpha_{H}-\alpha_{L}}$, i.e. $\frac{P_{H}}{\alpha_{H}}<\frac{P_{L}}{\alpha_{L}}$. Then $R_{H}=1-\frac{P_{H}}{\alpha_{H}}, R_{L}=0$, and the profit becomes $\pi_{P_{H}, P_{L}}\left(P_{H}, P_{L} \mid M=m, Q\right)=P_{H} \cdot \min \left(m \cdot R_{H}, Q\right)-\omega Q-\frac{1}{2} Q \varphi$. We assume that $Q$ is piecewise linear, the retailer has two optimal choice for $Q$.

$$
Q=0 \text { and } \pi_{P_{H}, P_{L}}^{1}\left(P_{H}, P_{L} \mid M=m, Q\right)=0 ;
$$


$Q=m \cdot R_{H}$ and $\pi_{P_{H}, P_{L}}^{1}\left(P_{H}, P_{L} \mid M=m, Q\right)=\left(P_{H}-\omega-\frac{1}{2} \varphi\right) \cdot m \cdot R_{H}=m\left(P_{H}-\omega-\frac{1}{2} \varphi\right)\left(1-\frac{P_{H}}{\alpha_{H}}\right)$, then I solve the profit function derivative on the high quality product price and I obtain $P_{H}=\frac{\left(\alpha_{H}+\omega+\frac{1}{2} \varphi\right)}{2}$, which results in a positive profit and is hence better than producing 0 units.

Senario 2: Suppose that $\frac{P_{H}}{\alpha_{H}} \leq \frac{P_{H}-P_{L}}{\alpha_{H}-\alpha_{L}}$, i.e. $\frac{P_{H}}{\alpha_{H}} \geq \frac{P_{L}}{\alpha_{L}}$, the retailer's profit is still piecewise linear in $Q$, therefore, she has three potentially optimal choices for $Q$ :

$$
Q=0, \text { and } \pi_{P_{H}, P_{L}}^{2}\left(P_{H}, P_{L} \mid M=m, Q\right)=0 ;
$$

$Q=m \cdot R_{H}$ and $\pi_{P_{H}, P_{L}}^{2}\left(P_{H}, P_{L} \mid M=m, Q\right)=\left(P_{H}-\omega-\frac{1}{2} \varphi\right) \cdot m \cdot \max \left(1-\frac{P_{H}-P_{L}}{\alpha_{H}-\alpha_{L}}, 0\right)$, suppose that $\frac{P_{H}-P_{L}}{\alpha_{H}-\alpha_{L}}>1$, we can get the same result as scenario 1; Suppose that $\frac{P_{H}-P_{L}}{\alpha_{H}-\alpha_{L}}<1$, so $\pi_{P_{H}, P_{L}}^{2}\left(P_{H}, P_{L} \mid M=m, Q\right)=\left(P_{H}-\omega-\frac{1}{2} \varphi\right) \cdot m\left(1-\frac{P_{H}-P_{L}}{\alpha_{H}-\alpha_{L}}\right)$. According to the presumption $\frac{P_{H}}{\alpha_{H}}<\frac{P_{H}-P_{L}}{\alpha_{H}-\alpha_{L}}$, then $\pi_{P_{H}, P_{L}}^{2}<\pi_{P_{H}, P_{L}}^{1}$, we do not take this case into consideration.

$$
\begin{aligned}
& Q=m \cdot\left(R_{H}+R_{L}\right)=m \cdot\left(1-\frac{P_{L}}{\alpha_{L}}\right) \text { and } \\
& \pi_{P_{H}, P_{L}}^{2}\left(P_{H}, P_{L} \mid M=m, Q\right)=m \cdot P_{H} \cdot\left(1-\frac{P_{H}-P_{L}}{\alpha_{H}-\alpha_{L}}\right)+m \cdot P_{L} \cdot\left(\frac{P_{H}-P_{L}}{\alpha_{H}-\alpha_{L}}-\frac{P_{L}}{\alpha_{L}}\right)-m \cdot\left(\omega+\frac{1}{2} \varphi\right)\left(1-\frac{P_{L}}{\alpha_{L}}\right), \text { so we only }
\end{aligned}
$$
consider case (iii). The retailer's optimization problem is now given by

$$
\begin{aligned}
& \max \pi_{P_{H}, P_{L}}^{2}\left(P_{H}, P_{L} \mid M=m, Q\right) \\
& =m \cdot P_{H} \cdot\left(1-\frac{P_{H}-P_{L}}{\alpha_{H}-\alpha_{L}}\right)+m \cdot P_{L} \cdot\left(\frac{P_{H}-P_{L}}{\alpha_{H}-\alpha_{L}}-\frac{P_{L}}{\alpha_{L}}\right)-m \cdot\left(\omega+\frac{1}{2} \varphi\right)\left(1-\frac{P_{L}}{\alpha_{L}}\right) \\
& \text { s.t. } \frac{P_{H}}{\alpha_{H}} \geq \frac{P_{L}}{\alpha_{L}} \quad P_{H}, P_{L}>0
\end{aligned}
$$

The Hessian for the objective function is given by:

$$
H=\left(\begin{array}{ll}
H_{1} & H_{2} \\
H_{3} & H_{4}
\end{array}\right)=\left(\begin{array}{cc}
\frac{-2 m}{\alpha_{H}-\alpha_{L}} & \frac{2 m}{\alpha_{H}-\alpha_{L}} \\
\frac{-2 m}{\alpha_{H}-\alpha_{L}}-\frac{2}{\alpha_{L}} & \frac{2 m}{\alpha_{H}-\alpha_{L}}
\end{array}\right)
$$

Which is negative definite $\left(H_{1}<0\right.$,

$\left.H_{2} \cdot H_{3}-H_{1} \cdot H_{4}=\frac{2 m}{\alpha_{H}-\alpha_{L}} \cdot\left(\frac{-2 m}{\alpha_{H}-\alpha_{L}}-\frac{2}{\alpha_{L}}\right)-\frac{4 m^{2}}{\left(\alpha_{H}-\alpha_{L}\right)^{2}}=\frac{-4 m}{\alpha_{L} \cdot\left(\alpha_{H}-\alpha_{L}\right)}<0\right)$. Because of the constraint condition, we need construct Lagrange function $L\left(P_{H}, P_{L}, \sigma\right)=m \cdot P_{H} \cdot\left(1-\frac{P_{H}-P_{L}}{\alpha_{H}-\alpha_{L}}\right)+m \cdot P_{L} \cdot\left(\frac{P_{H}-P_{L}}{\alpha_{H}-\alpha_{L}}-\frac{P_{L}}{\alpha_{L}}\right)-m \cdot\left(\omega+\frac{1}{2} \varphi\right)\left(1-\frac{P_{L}}{\alpha_{L}}\right)+\sigma\left(\frac{P_{H}}{\alpha_{H}}-\frac{P_{L}}{\alpha_{L}}\right)$. Therefore, KKT conditions are necessary and sufficient and are given by: 


$$
\left\{\begin{array}{l}
\frac{\partial \pi}{\partial P_{H}}=m\left(1-2\left(\frac{P_{H}-P_{L}}{\alpha_{H}-\alpha_{L}}\right)\right)+\frac{\sigma}{\alpha_{H}} \leq 0, P_{H}>0, P_{H} \frac{\partial \pi}{\partial P_{H}}=0 \\
\frac{\partial \pi}{\partial P_{L}}=m\left(2\left(\frac{P_{H}-P_{L}}{\alpha_{H}-\alpha_{L}}\right)+\frac{\omega+\frac{1}{2} \varphi-2 P_{L}}{\alpha_{L}}\right)-\frac{\sigma}{\alpha_{L}} \leq 0, P_{L}>0, P_{L} \frac{\partial \pi}{\partial P_{L}}=0 \\
\frac{\partial \pi}{\partial \sigma}=\frac{P_{H}}{\alpha_{H}}-\frac{P_{L}}{\alpha_{L}} \geq 0, \sigma \geq 0, \sigma \frac{\partial \pi}{\partial \sigma}=0
\end{array}\right.
$$

Suppose that $\sigma=0$, we obtain $P_{L}=\frac{\omega+\frac{1}{2} \varphi+\alpha_{L}}{2}$ and $P_{H}=\frac{\omega+\frac{1}{2} \varphi+\alpha_{H}}{2}$. However, we find these values are not feasible because $\frac{P_{H}}{\alpha_{H}}>\frac{P_{L}}{\alpha_{L}}$. Therefore, suppose that $\sigma>0$, and hence $\frac{\partial \pi}{\partial \sigma}=\frac{P_{H}}{\alpha_{H}}-\frac{P_{L}}{\alpha_{L}}=0$, i.e. $\frac{P_{H}}{\alpha_{H}}=\frac{P_{L}}{\alpha_{L}}$. And the retailer's profit becomes $\pi_{P_{H}, P_{L}}\left(P_{H}, P_{L} \mid M=m, Q\right)=m\left(P_{H}-\omega-\frac{1}{2} \varphi\right)\left(1-\frac{P_{H}}{\alpha_{H}}\right)$. We have the optimal pricing and ordering quantity of high and low quality fresh product:

$$
\left\{\begin{array}{l}
P_{H}=\frac{\omega+\frac{1}{2} \varphi+\alpha_{H}}{2} \\
P_{L}=\frac{\omega+\frac{1}{2} \varphi+\alpha_{L}}{2} \\
Q=\frac{\left(\alpha_{H}-\left(\omega+\frac{1}{2} \varphi\right)\right)}{2 \alpha_{H}} m \\
\pi_{P_{H}, P_{L}}\left(P_{H}, P_{L} \mid M=m, Q\right)=\frac{\left(\alpha_{H}-\left(\omega+\frac{1}{2} \varphi\right)\right)^{2}}{4 \alpha_{H}} m
\end{array}\right.
$$

\section{Numerical Analysis}

Giving a fresh product as an example, we assume that $u$ is a customer's quality sensitivity, $u \sim U[0,1]$. There are discussions about the optimal decision of retailer and fluctuation of the potential demand for market. We have assumptions about some values of parameters as following tables (Table 1 and Table 2).

Proposition 1: Facing the two quality levels of fresh products, retailers will not benefit from sales of lower quality of fresh products with the deterministic demand.

Proof 1: From the optimal retailer's profit $\frac{\left(\alpha_{H}-\left(\omega+\frac{1}{2} \varphi\right)\right)^{2}}{4 \alpha_{H}} m$, we can infer that the retailer's profit is only related to high quality factor, ordering and preserving costs. This is because retailer sells low quality products at a discount price, which still needs to undertake ordering and preserving costs. Therefore, the retailer sells low quality fresh products only to minimum the loss and does not make profit from it.

Proposition 2: To realize the optimal profit, the retailer's initial ordering quantity is smaller than the potential demand for market. 
Table 1. Value of parameters.

\begin{tabular}{cccc}
\hline$\alpha_{L}$ & $\alpha_{H}$ & $\omega$ & $\varphi$ \\
\hline & 1.5 & & 0.2 \\
& 2.5 & 1 & \\
\hline
\end{tabular}

Table 2. Sensitivity analysis.

\begin{tabular}{ccccc}
\hline$m$ & $Q$ & $P_{H}$ & $P_{L}$ & $\pi_{P_{H}, P_{L}}$ \\
\hline \multirow{2}{*}{100} & 13.3 & 1.3 & & 2.7 \\
& 28 & 1.8 & 0.7 & 19.6 \\
& 34.3 & 2.3 & 41 & 5.4 \\
200 & 26.6 & 1.3 & 39.2 & 82 \\
& 56 & 1.8 & & 7.1 \\
& 68.2 & 2.3 & & 58.8 \\
\hline
\end{tabular}

Proof 2: From $Q=\frac{\left(\alpha_{H}-\left(\omega+\frac{1}{2} \varphi\right)\right)}{2 \alpha_{H}} m$, we can obtain $0<\frac{\left(\alpha_{H}-\left(\omega+\frac{1}{2} \varphi\right)\right)}{2 \alpha_{H}}<1$, i.e. $Q<m$. This is mainly because the retailer sells high quality fresh product at a higher price to make more profit. As long as the retailer has low ordering and preservation costs, it will have the motive to repeat order, which is consistent with zero inventory and rapid inventory turnover in operation management.

\section{Conclusion and Future Research}

This paper focuses on multiple quality fresh products and considers consumer utility to analyze the retailer's optimal ordering and pricing strategies. Our results showed that, facing the two quality levels of fresh products, retailers would not benefit from sales of lower quality of fresh products with the deterministic demand. In the pursuit of profit maximization, the initial order quantity is smaller than the potential demand for market. Possible extensions of this paper involve relaxing some of assumptions, for example, considering random customer arrival process and demand substitution of high and low quality products.

\section{References}

[1] Goyal, S.K. and Giri, B.C. (2001) Recent Trends in Modeling of Deteriorating Inventory. European Journal of Operational Research, 134, 1-16. http://dx.doi.org/10.1016/S0377-2217(00)00248-4

[2] Li, R., Lan, H. and Mawhinney, J.R. (2010) A Review on Deteriorating Inventory Study. Journal of Service Science and Management, 3, 117-129. http://dx.doi.org/10.4236/jssm.2010.31015

[3] Bakker, M., Riezebos, J. and Teunter, R.H. (2012) Review of Inventory Systems with Deterioration since 2001. European Journal of Operational Research, 221, 275-284. http://dx.doi.org/10.1016/j.ejor.2012.03.004

[4] Xiao, Y., Wu, P. and Wang, Y. (2010) Pricing Strategies for Fresh Products with Multiple Quality Levels Based on Customer Choice Behavior. Chinese Journal of Management Science, 18, 58-65.

[5] Bai, R. and Kendall, G. (2008) A Model for Fresh Produce Shelf-Space Allocation and Inventory Management with Freshness-Condition-Dependent Demand. INFORMS Journal on Computing, 20, 78-85.

http://dx.doi.org/10.1287/ijoc.1070.0219 
[6] Tal, A. and Teijo, A. (2009) An EOQ Model for Items with a Fixed Shelf-Life and a Declining Demand Rate Based on Time-to-Expiry Technical Note. Asia-Pacific Journal of Operational Research, 26, 759-767. http://dx.doi.org/10.1142/S0217595909002456

[7] Dan, B. and Chen, J. (2008) Coordinating Fresh Agricultural Supply Chain under the Valuable Loss. Chinese Journal of Management Science, $\mathbf{5}, 6$.

[8] Cai, X., Chen, J., Xiao, Y., et al. (2010) Optimization and Coordination of Fresh Product Supply Chains with Freshness-Keeping Effort. Production and Operations Management, 19, 261-278. http://dx.doi.org/10.1111/j.1937-5956.2009.01096.x

[9] Ferguson, M.E. and Koenigsberg, O. (2007) How Should a Firm Manage Deteriorating Inventory? Production and Operations Management, 16, 306-321. http://dx.doi.org/10.1111/j.1937-5956.2007.tb00261.x

[10] Akcay, Y., Natarajan, H.P. and Xu, S.H. (2010) Joint Dynamic Pricing of Multiple Perishable Products under Consumer Choice. Management Science, 56, 1345-1361. http://dx.doi.org/10.1287/mnsc.1100.1178

[11] Li, Y., Cheang, B. and Lim A. (2012) Grocery Perishables Management. Production and Operations Management, 21, 504-517. http://dx.doi.org/10.1111/j.1937-5956.2011.01288.x

[12] Sainathan, A. (2013) Pricing and Replenishment of Competing Perishable Product Variants under Dynamic Demand Substitution. Production and Operations Management, 22, 1157-1181. 